\title{
Substratos alternativos ao xaxim no cultivo do híbrido primário Miltonia regnellii Rchb. f. X Oncidium concolor Hook. (Orchidaceae)
}

\author{
Alternative substrates to substitute xaxim in the cultivation of the \\ primary hybrid Miltonia regnellii Rchb. f. X Oncidium concolor \\ Hook. (Orchidaceae)
}

\author{
Lilian Yukari Yamamoto후 Mauren Sorace ${ }^{2}$; Ricardo Tadeu Faria ${ }^{3 *}$; \\ Lúcia Sadayo Takahashi ${ }^{4}$; Jenniffer Aparecida Schnitzer ${ }^{5}$
}

\section{Resumo}

\begin{abstract}
O presente trabalho teve como objetivo avaliar substratos alternativos ao xaxim, no desenvolvimento vegetativo e radicular de plantas aclimatizadas do híbrido primário de orquídea Miltonia regnellii Rchb. f. X Oncidium concolor Hook. Mudas com aproximadamente oito meses, obtidas por propagação in vitro e com altura média $6,4 \pm 0,5 \mathrm{~cm}$, foram cultivadas em casa de vegetação com $60 \%$ de sombreamento em vasos de polipropileno número um, contendo os seguintes substratos: $\mathrm{XD}=$ xaxim desfibrado (controle); $\mathrm{PBC}=$ pó de bagaço de cana-de-açúcar; $\mathrm{PBC}: \mathrm{I}=$ pó de bagaço de cana-de-açúcar: isopor picado $(1: 1$, $\mathrm{v}: \mathrm{v}) ; \mathrm{PBC}: \mathrm{E}=$ pó de bagaço de cana-de-açúcar: esfagno $(1: 1, \mathrm{v}: \mathrm{v}) ; \mathrm{PBC}: \mathrm{CP}=$ pó de bagaço de canade-açúcar: casca de pínus $(1: 1, \mathrm{v}: \mathrm{v}) ; \mathrm{PC}=$ pó de coco e $\mathrm{PBC}: \mathrm{PC}=$ pó de bagaço de cana-de-açúcar: pó de coco $(1: 1, \mathrm{v}: \mathrm{v})$. O delineamento experimental utilizado foi de blocos casualizados com 10 plantas por tratamento. As variáveis avaliadas após 12 meses do início do experimento foram: altura da parte aérea (APA), comprimento do maior pseudobulbo (CMP), diâmetro do maior pseudobulbo (DMP), comprimento da maior raiz (CMR), número de raízes (NR), número de brotos $(\mathrm{NB})$, massa fresca total (MFT) e pH do substrato. Todos os substratos avaliados, com exceção do pó de bagaço de cana-deaçúcar: esfagno podem ser utilizados como substitutos ao xaxim no cultivo do híbrido Miltonia regnellii Rchb. f. X Oncidium concolor Hook.

Palavras-chave: Orquídeas, floricultura, Dicksonia sellowiana Hook, preservação
\end{abstract}

\begin{abstract}
The paper had the purpose of evaluating root formation and vegetative development of the acclimatized plants of the primary hybrid Miltonia regnellii Rchb. f. X Oncidium concolor Hook., using alternative substrates to substitute xaxim. Seedlings, with approximately eight months, obtained by in vitro culture with medium height of $6.4 \pm 0.5 \mathrm{~cm}$, were grown up in greenhouse with $60 \%$ of shadowness, using pots of polipropilene number one, containing the following substrates as treatments: $\mathrm{XD}=$ defibered xaxim (control); $\mathrm{PBC}=$ sugar cane bagasse powder; $\mathrm{PBC}: \mathrm{I}=$ sugar cane bagasse powder: estruted polystyrene (1:1, v:v); PBC:E= sugar cane bagasse powder: sphagnum (1:1, v:v); PBC:PC = sugar cane bagasse powder: husk pinus $(1: 1, \mathrm{v}: \mathrm{v}) ; \mathrm{CP}=$ coconut powder and $\mathrm{PBC}: \mathrm{PC}=$ coconut powder: sugar cane bagasse powder $(1: 1, \mathrm{v}: \mathrm{v})$. The experimental design was randomized blocks,
\end{abstract}

1 Engenheira Agrônoma. E-mail: yamamochiyukari@yahoo.com.br.

2 Doutoranda em Agronomia da UEL. Bolsista Capes.

3 Eng ${ }^{\circ}$ Agr., Dr., Professor Associado do Departamento de Agronomia da UEL. Londrina, PR. E-mail: faria@uel.br

4 Eng $^{\circ}$ Agr., Dra., Professora Associado do Departamento de Agronomia da UEL.

5 Mestranda em Agronomia da UEL.

* Autor para correspondência 
with seven treatments, and ten replications. Growing parameters evaluated after 12 months were: height of above ground part (APA), length of the heigher pseudobulb (CMP), diameter of the heigher pseudobulb (DMP), length of the heigher root (CMR), number of roots (NR), number of buds (NB), total fresh mass weight (MFT) and substrate $\mathrm{pH}$. All the studied substrates, except the sugar cane bagasse powder with sphagnum, can be recommended to substitute xaxim on the hybrid Miltonia regnellii Rchb. f. X Oncidium concolor Hook. crop.

Key words: Orchids, floriculture, Dicksonia sellowiana Hook, preservation

\section{Introdução}

A família Orchidaceae é composta por aproximadamente 35.000 espécies, distribuídas em mais de 600 gêneros distintos, e vários híbridos, obtidos mediante cruzamentos interespecíficos e/ou intergenéricos, possuindo uma grande variabilidade de formas, tamanhos e cores de folhas e flores. A grande maioria das espécies encontra-se em regiões tropicais, embora existam representantes em regiões temperadas e até mesmo alguns exemplares em regiões boreais (SUTTLEWORTH et al., 1970; SILVA, 1986; HUMBER, 1994; PASCAL et al., 2005).

O substrato é o meio onde se desenvolvem as raízes das plantas cultivadas fora do solo, servindo como suporte e podem ser fonte de nutrientes. O substrato pode ser formado de solo mineral ou orgânico e de um só ou de diversos materiais em misturas (SILVA; SILVA, 1997; KÄMPF, 2000).

Devido ao fato do xaxim (Dicksonia sellowiana Hook.), que era utilizado como substrato para orquídeas, encontrar-se em risco de extinção devido ao constante extrativismo, vários estudos vêm sendo realizados visando o uso de substratos alternativos. Por se tratar de uma espécie de crescimento muito lento, essa planta deve ser protegida e o seu uso desestimulado. Em 1992, devido ao intenso extrativismo, foi criada no Rio Grande do Sul, a lei 9.519, que proíbe a extração do xaxim em florestas nativas (KÄMPF, 2000).

De modo geral, observa-se que diferentes tipos de resíduos agroindustriais vêm sendo progressivamente aplicados como substrato (casca de arroz, bagaço de cana, casca de pínus, etc.), visando oferecer alternativas para produtores de mudas e minimizar o impacto ambiental provocado pelos resíduos sólidos gerados pela agroindústria. Ultimamente, tem-se verificado a introdução de cultivos agrícolas em substratos à base de casca de coco seco, substituindo outros materiais, tais como: lã de rocha, turfas de esfagno, areia, etc. (ROSA et al., 2002). A utilização de resíduos da agroindústria disponíveis regionalmente como componente para substratos pode propiciar a redução de custos de produção e transporte, assim como auxiliar na minimização da poluição decorrente do acúmulo desses materiais no meio ambiente (FERMINO, 1996). Resíduos agrícolas como fibra de coco, casca de pínus, casca de arroz carbonizada são exemplos de substratos utilizados como alternativos ao xaxim na floricultura. De acordo com a literatura estes substratos foram avaliados por alguns autores tais como: Kämpf (1999), Faria et al. (2001), Rosa et al. (2001), Souza e Jasmim (2001), Bosa et al. (2003), Guerrini e Trigueiro (2004), Meneguce, Oliveira e Faria (2004) e muitos outros.

O bagaço de cana-de-açúcar tem um potencial de aproveitamento, podendo ser utilizado na alimentação animal, na adubação, e na fabricação de vários tipos de papéis, e de produtos com aplicação na indústria química e farmacêutica. Pode ser utilizado também na obtenção de plástico biodegradável e para a geração de energia (energia elétrica da biomassa), garantindo a energia consumida pela própria usina de beneficiamento e obtendo excedente comercializável (NEGRÃO; URBAN, 2005).

O presente trabalho teve como objetivo avaliar substratos alternativos ao xaxim no desenvolvimento vegetativo e radicular de plantas do híbrido primário 
Miltonia regnellii Rchb. f. X Oncidium concolor Hook., com oito meses de idade.

\section{Material e métodos}

O experimento foi conduzido no Centro de Ciências Agrárias da Universidade Estadual de Londrina (UEL), Paraná, no período de abril de 2006 a março de 2007.

Foram utilizadas mudas aclimatizadas do híbrido primário obtido a partir do cruzamento de Miltonia regnellii Rchb. f. X Oncidium concolor Hook., com oito meses de idade e 6,4 $\pm 0,5 \mathrm{~cm}$ de altura, as quais foram obtidas por semeadura in vitro. Os vasos contendo as plantas foram mantidos em bancadas suspensas, em casa de vegetação, com proteção de tela de polipropileno de coloração preta, com retenção de $60 \%$ do fluxo de radiação solar. A temperatura média durante o período do experimento foi de $25 \pm 5^{\circ} \mathrm{C}$ e a umidade relativa de 50 a $60 \%$, clima subtropical (CFA).

Os recipientes utilizados foram vasos de plástico (polipropileno), número um, de coloração preta, com $12,0 \mathrm{~cm}$ de altura, $8,5 \mathrm{~cm}$ de diâmetro e quatro orifícios na base. Para favorecer a drenagem e a aeração do sistema radicular foi acondicionada uma camada de pedra brita número dois no fundo dos vasos.

Os tratamentos consistiram dos seguintes substratos: $\mathrm{XD}=$ xaxim desfibrado (testemunha); $\mathrm{PBC}=$ pó de bagaço de cana-de-açúcar (Pó Eco Vegetal-Santa Rita, patente $\mathrm{n}^{\circ}$ - 0.403.527-5); PBC:I= pó de bagaço de cana-de-açúcar: isopor picado, com granulometria média de 1 a $2 \mathrm{~cm}$ de diâmetro; $\mathrm{PBC}: \mathrm{E}=$ pó de bagaço de cana-de-açúcar: esfagno; $\mathrm{PBC}: \mathrm{PC}=$ pó de bagaço de cana-de-açúcar: casca de pínus, com granulometria média de $1 \mathrm{~cm}$ de diâmetro; $\mathrm{PC}=$ pó de coco (Padrão 11-Amafibra $\left.{ }^{\circledR}\right)$ e PBC:PC= pó de bagaço de cana-de-açúcar: pó de coco (Padrão 11-Amafibra ${ }^{\circledR}$ ). Os substratos foram misturados em proporções volumétricas iguais.

A irrigação foi realizada através de uma mangueira, com freqüência de uma vez por semana durante o inverno e duas vezes por semana durante o verão. As plantas foram adubadas quinzenalmente através de fertirrigação com adubo químico de formulação NPK (20:20:20) na concentração de $1 \mathrm{~g}$. $\mathrm{L}^{-1}$, utilizando $30 \mathrm{~mL}$. $\mathrm{L}^{-1}$ por vaso.

O experimento foi avaliado após 12 meses da instalação, sendo analisadas as seguintes variáveis: altura da parte aérea (APA), comprimento do maior pseudobulbo (CMP), diâmetro do maior pseudobulbo (DMP), comprimento da maior raiz (CMR), número de raízes (NR), número de brotos (NB), massa fresca total (MFT) e pH do substrato.

O delineamento experimental utilizado foi de blocos casualizados, com 10 plantas por tratamento, contendouma planta porvaso. Os dados obtidos foram submetidos à análise de variância complementadas pelo teste de Tukey à $5 \%$ de significância. Para as variáveis número de raízes (NR) e número de brotos (NB), os dados foram transformados pela constante $\sqrt{\mathrm{x}+1}$, sendo que as médias apresentadas não são as transformadas.

\section{Resultados e discussão}

Os resultados referentes à altura da planta, comprimento do pseudobulbo, diâmetro do pseudobulbo, comprimento da maior raiz, número de raízes, número de brotos e massa fresca total de plantas de Miltonia regnellii Rchb. f. X Oncidium concolor Hook, são apresentados na Tabela 1. 
Tabela 1- Média dos tratamentos referentes aos parâmetros: altura da parte aérea (APA), comprimento do maior pseudobulbo (CMP), diâmetro do maior pseudobulbo (DMP), comprimento da maior raiz (CMR), número de raízes (NR), número de brotos (NB) e massa fresca total (MFT) de plantas aclimatizadas, com oito meses, de Miltonia regnellii Rchb. f. X Oncidium concolor Hook., após 12 meses do início do experimento. UEL, Londrina-PR, 2007.

\begin{tabular}{cccccccc}
\hline Tratamento* & APA $(\mathrm{cm})$ & CMP $(\mathrm{cm})$ & DMP $(\mathrm{cm})$ & CMR $(\mathrm{cm})$ & NR** & NB** & MFT $(\mathrm{g})$ \\
\hline XD & $15,75 \mathrm{ab}$ a $^{* *}$ & $5,31 \mathrm{ab}$ & $1,99 \mathrm{ab}$ & $17,18 \mathrm{abc}$ & $53,50 \mathrm{a}$ & $5,60 \mathrm{ab}$ & $27,25 \mathrm{ab}$ \\
PBC & $16,04 \mathrm{a}$ & $6,23 \mathrm{a}$ & $2,25 \mathrm{a}$ & $18,90 \mathrm{ab}$ & $45,70 \mathrm{a}$ & $5,70 \mathrm{ab}$ & $33,21 \mathrm{a}$ \\
PBC:I & $16,38 \mathrm{a}$ & $5,96 \mathrm{a}$ & $2,09 \mathrm{ab}$ & $21,50 \mathrm{a}$ & $47,10 \mathrm{a}$ & $7,40 \mathrm{a}$ & $33,89 \mathrm{a}$ \\
PBC:E & $12,26 \mathrm{~b}$ & $4,85 \mathrm{~b}$ & $1,84 \mathrm{~b}$ & $12,17 \mathrm{c}$ & $26,33 \mathrm{~b}$ & $3,78 \mathrm{~b}$ & $17,69 \mathrm{~b}$ \\
PBC:CP & $14,7 \mathrm{ab}$ & $5,44 \mathrm{ab}$ & $1,91 \mathrm{ab}$ & $19,00 \mathrm{ab}$ & $37,22 \mathrm{ab}$ & $6,25 \mathrm{a}$ & $39,31 \mathrm{a}$ \\
PC & $14,94 \mathrm{ab}$ & $5,90 \mathrm{a}$ & $2,07 \mathrm{ab}$ & $19,95 \mathrm{ab}$ & $41,80 \mathrm{a}$ & $5,56 \mathrm{ab}$ & $27,56 \mathrm{ab}$ \\
PBC:PC & $15,70 \mathrm{ab}$ & $5,43 \mathrm{ab}$ & $2,00 \mathrm{ab}$ & $19,60 \mathrm{ab}$ & $50,80 \mathrm{a}$ & $7,50 \mathrm{a}$ & $36,06 \mathrm{a}$ \\
\hline CV $(\%)$ & 17,76 & 13,05 & 13,21 & 21,85 & 15,34 & 13,14 & 15,51 \\
\hline
\end{tabular}

${ }^{*} \mathrm{XD}=$ xaxim desfibrado (controle); $\mathrm{PBC}=$ pó de bagaço de cana-de-açúcar; $\mathrm{PBC}: \mathrm{I}=$ pó de bagaço de cana-de-açúcar: isopor picado; $\mathrm{PBC}: \mathrm{E}=$ pó de bagaço de cana-de-açúcar: esfagno; $\mathrm{PBC}: \mathrm{CP}=$ pó de bagaço de cana-de-açúcar: casca de pinus; $\mathrm{PC}=$ pó de coco; $\mathrm{PBC}: \mathrm{PC}=$ pó de bagaço de cana-de-açúcar: pó de coco.

${ }^{* *}$ Dados sob tranformação $\sqrt{\mathrm{x}+1}$.

${ }^{* * *}$ Médias seguidas da mesma letra na vertical não diferem entre si pelo teste de Tukey ao nível de 5\% de significância.

Os resultados demonstraram que para a variável altura da parte aérea os tratamentos $\mathrm{PBC}$ e PBC:I obtiveram os maiores valores, mas não diferiram estatisticamente da testemunha XD e dos tratamentos que tinham $\mathrm{CP}$ e $\mathrm{PC}$, e apresentaram médias superiores em relação à mistura com esfagno. Yamakami et al. (2006), cultivando um híbrido de Cattleya em diferentes substratos, obtiveram melhores resultados para a altura da parte aérea utilizando a fibra de coco, casca de pínus pura e em combinação com casca de arroz carbonizada em diferentes proporções volumétricas $(1: 1, \mathrm{v}: \mathrm{v}$; $2: 1, \mathrm{v}: \mathrm{v} ; 1: 2, \mathrm{v}: \mathrm{v})$.

Assis et al. (2005) obtiveram um bom desenvolvimento da parte aérea de Dendrobium nobile Lindl quando utilizaram coco em pó como substrato alternativo ao xaxim. Moraes, Cavalcante e Faria (2002) obtiveram bons resultados utilizando a mistura de plantmax + carvão vegetal + isopor moído (1:1:1, v:v), para aclimatização da mesma espécie. Contudo, Stegani (2006), em seu trabalho de aclimatização Miltonia regnellii Rchb. f. X Oncidium concolor Hook., não observou diferenças significativas para a característica altura da planta entre todos esses mesmos substratos aqui testados e a mistura composta de vermiculita + Plantimax $^{\circledR}$ $(1: 1, v: v)$.

Para a variável comprimento do maior pseudobulbo, os tratamentos PBC, PBC:I e PBC:PC apresentaram as maiores médias, com 6,23, 5,96 e 5,9 $\mathrm{cm}$, respectivamente, e diferiram estatisticamente da mistura com esfagno que apresentou a menor média $(4,85 \mathrm{~cm})$. No entanto, não diferiram da testemunha (XD) e dos tratamentos PC e CP. Yamakami et al. (2006), observaram resultados satisfatórios para o comprimento do maior pseudobulbo, quando cultivaram o híbrido de Cattleya nos substratos fibra de coco e casca de pínus pura ou em combinação com casca de arroz carbonizada em diferentes proporções (1:1, v:v; 2:1,v:v; 1:2, v:v).

Para a variável diâmetro do maior pseudobulbo, a mistura com esfagno foi inferior estatisticamente ao PBC puro, que apresentou o maior diâmetro $(2,25 \mathrm{~cm})$, porém estes não diferiram em relação aos demais substratos testados. Estudos realizados por Moraes et al. (2002), utilizando a mistura de Plantmax $^{\circledR}+$ carvão vegetal + isopor moído (1:1:1, v:v), e por Assis et al. (2005), testando coco em pó, no cultivo de Dendrobium nobile Lindl., demonstraram um bom desenvolvimento 
do diâmetro do pseudobulbo. De acordo com Assis et al. (2003), o pseudobulbo exerce um importante papel na demanda energética da planta, uma vez que armazena água e carboidratos.

Quanto à variável comprimento da maior raiz, a mistura com esfagno apresentou a menor média $(13,1 \mathrm{~cm})$ e diferiu estatisticamente em relação aos tratamentos PBC, PBC:I, PBC:CP, PC, PBC:CP que apresentaram as maiores médias. Stegani (2006), estudando os mesmos substratos como alternativos ao xaxim para a aclimatização de plântulas do híbrido Miltonia regnellii Rchb. f. X Oncidium concolor Hook., observou valores superiores de comprimento da maior raiz para os substratos pó de coco e mistura de pó de bagaço de cana-de-açúcar com o pó de coco (1:1, v:v). Assis et al. (2005), no cultivo de Dendrobium nobile Lindl., obtiveram bons resultados utilizando coco em pó em substituição ao xaxim.

Com relação ao número de raízes, a testemunha (XD) e os tratamentos PBC, PBC:I, PC e PBC: PC obtiveram os maiores valores em relação à mistura com esfagno. Resultados semelhantes foram observados por Moraes et al. (2002), estudando diferentes substratos para aclimatização de Dendrobium nobile Lindl., observaram bom número de raízes utilizando a mistura de plantmax + carvão vegetal + isopor moído (1:1:1, v:v:v). No cultivo do híbrido de Cattleya utilizando substratos alternativos ao xaxim, Yamakami et al. (2006) concluíram que a fibra de coco favorece o desenvolvimento de mais raízes, enquanto que a casca de pínus pura não demonstrou eficiência.

Quanto ao número de brotos, os tratamentos PBC:I $(7,4)$, PBC:CP $(6,25)$ e PBC:PC $(7,5)$ foram superiores em relação à mistura com esfagno $(3,78)$, porém não diferiram estatisticamente dos demais tratamentos. Demattê (2001) obteve resultados satisfatórios com relação ao número de brotos da bromélia epifita Tillandsia gardneri, utilizando misturas à base de coco. Galdiano Júnior, Moraes e Lemos (2007), verificaram que o substrato constituído de casca de pínus + carvão $(1: 1, \mathrm{v}: \mathrm{v})$ apresentou baixa eficiência, quanto ao número de brotos, sendo que a fibra de coco, o esfagno e o coco em pedaços, respectivamente, proporcionaram um maior crescimento do híbrido de Cattleya Lindl. Neste caso, o esfagno ao ser diluído numa mistura com mais condicionadores, teve sua ação potencializada.

Rego et al. (2000), trabalhando com as espécies Oncidium sarcodes e Schomburgkia crispa, concluíram que é possível a substituição do xaxim por misturas de casca de pínus, isopor, carvão vegetal, vermiculita e casca de arroz carbonizada, sem prejudicar o crescimento da parte aérea e as brotações. Basso e Faria (2002), conseguiram bons resultados para produção de brotos de Laelia lundii cultivada em substrato de esfagno misturado com fibra de coco.

Para a variável massa fresca total da planta, a mistura com esfagno apresentou a menor média $(17,69 \mathrm{~g})$, sendo diferente estatisticamente dos tratamentos com bagaço-de-cana (PBC, PBC:I, PBC:CP, PBC:PC), que apresentaram as maiores médias $(33,21,33,89,39,31$ e 36,06 $\mathrm{g}$, respectivamente), mas que não diferiram da testemunha (XD) e do pó de coco (PC). Yamakami et al. (2006), avaliando o cultivo do híbrido de Cattleya (Lindl.) (Orchidaceae) em substratos alternativos ao xaxim, obtiveram bons resultados em relação à massa fresca total, utilizando a fibra de coco pura e a casca de pínus + casca de arroz carbonizada (2:1, v:v). Colombo et al. (2005), trabalhando com diferentes substratos na aclimatização da orquídea Cattleya chocolate drop X (C. guttata X L. tenebrosa), não obtiveram bons resultados quanto à massa fresca da planta utilizando esfagno, enquanto o substrato pó de coco foi o mais eficiente, concordando com este trabalho.

De acordo com os dados da Figura 1, observa-se que o $\mathrm{pH}$ dos substratos avaliados após 12 meses do início do experimento apresentaram valores de 6,3 a 7,1. Os valores mais baixos de $\mathrm{pH}$ foram para o tratamento $\mathrm{PBC}: \mathrm{CP}(6,33)$ seguido da testemunha (XD) $(6,44)$. Assis et al. (2005) obtiveram valores 
próximos de $\mathrm{pH}$ para o xaxim desfibrado $(6,19)$ no cultivo de Dendrobium nobile Lindl. (Orchidaceae), após oito meses do início do experimento. Contudo, Yamakami et al. (2006) obtiveram resultados mais baixo de $\mathrm{pH}$ para o xaxim $(4,0)$ durante doze meses do cultivo da orquídea Cattleya labiata X Cattleya forbesii. Kämpf (2000) relata $\mathrm{pH}$ em torno de 4,0 a 4,2 para fibra de xaxim e 4,0 a 4,5 para casca de pínus. Bezerra et al. (2001) e Lacerda et al. (2006), trabalhando com pó de coco, obtiveram valor de $\mathrm{pH}$ de 6,7 e 6,3 respectivamente. Estes valores foram semelhantes aos obtidos neste trabalho para o substrato pó de coco.

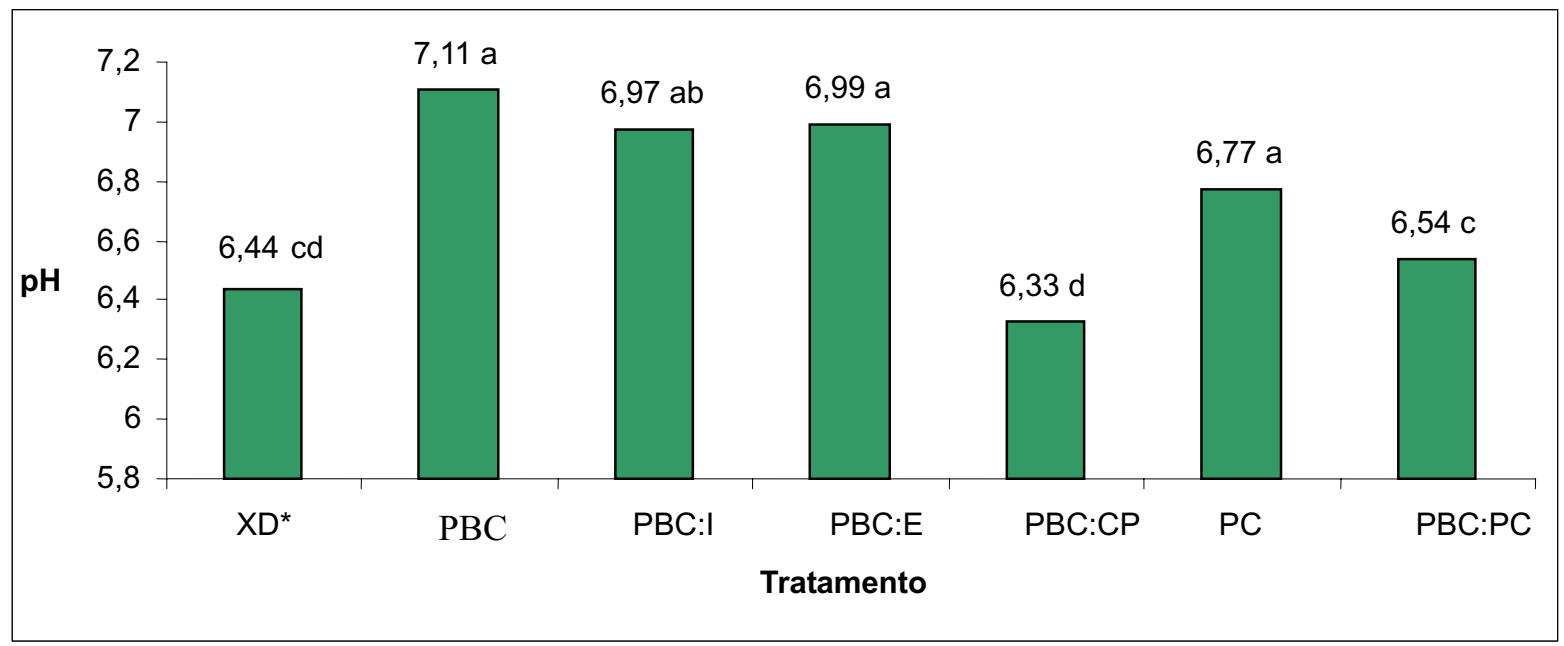

Figura 1. Valores médios do potencial hidrogeniônico $(\mathrm{pH})$ nos diferentes substratos, utilizados no cultivo de plantas aclimatizadas de Miltonia regnellii Rchb. f. X Oncidium concolor Hook., após 12 meses do início do experimento. UEL, Londrina-PR, 2007.

${ }^{*} \mathrm{XD}=$ xaxim desfibrado (controle); $\mathrm{PBC}=$ pó de bagaço de cana-de-açúcar; $\mathrm{PBC}: \mathrm{I}=$ pó de bagaço de cana-de-açúcar: isopor picado; $\mathrm{PBC}: \mathrm{E}=$ pó de bagaço de cana-de-açúcar: esfagno; $\mathrm{PBC}: \mathrm{CP}=$ pó de bagaço de cana-de-açúcar: casca de pinus; $\mathrm{PC}=$ pó de coco; $\mathrm{PBC}: \mathrm{PC}=$ pó de bagaço de cana-de-açúcar: pó de coco.

O monitoramento do $\mathrm{pH}$ é importante, pois ele tem influência na disponibilidade de nutrientes e nos processos fisiológicos da planta. Recomenda-se que para plantas ornamentais o $\mathrm{pH}$ deve estar na faixa de 5,0 a 5,8 em substratos onde predomina a matéria orgânica, e entre 6,0 e 6,5, quando for à base de solo mineral. O pH final nas misturas dos substratos vai depender da capacidade de tamponamento de cada um dos substratos e suas interações (KÄMPF, 2000). Segundo Röber e Schaller (1985 apud KÄMPF, 2000), para as orquídeas do gênero Oncidium, o pH ideal está na faixa de 5,5 a 6,5. Apesar dos tratamentos PBC, PBC:I, PC e PBC:PC apresentarem valores de $\mathrm{pH}$ do substrato superiores à faixa ideal, este fator não interferiu no bom desenvolvimento de mudas do híbrido Miltonia regnellii Rchb. f. X Oncidium concolor Hook durante um ano de cultivo.

Não foram observadas diferenças significativas entre o xaxim desfibrado e os demais substratos testados. A mistura PBC com esfagno apresentou as médias mais baixas em todos os parâmetros avaliados, provavelmente por ter ocorrido uma maior retenção de água neste substrato (KÄMPF, 2000), prejudicando o cultivo durante um ano das plantas de orquídea aclimatizadas (oito meses) utilizadas nesse trabalho.

Stegani (2006) estudando a aclimatização dessa mesma orquídea, concluiu que somente o pó de bagaço de cana-de-açúcar na mistura com o pó de 
coco $(1: 1, \mathrm{v}: \mathrm{v})$ e o pó de coco puro foram os mais indicados para substituir o xaxim, durante a etapa de aclimatização, enquanto que no presente trabalho, além dos substratos mencionados, pode-se utilizar o pó de bagaço de cana-de-açúcar, puro e em misturas com isopor picado $(1: 1, \mathrm{v}: \mathrm{v})$, e com casca de pínus (1:1, v:v). Esta diferença pode ser explicada pelo fato das mudas durante a aclimatização serem mais sensíveis com relação ao tipo de substrato do que as plantas em desenvolvimento.

\section{Conclusão}

Os substratos pó de coco, pó de bagaço de canade-açúcar puro ou na mistura em proporções iguais com outros materiais como: isopor picado, casca de pínus e o pó do coco podem ser utilizados em substituição ao xaxim, com exceção da sua mistura com esfagno que não demonstrou resultados favoráveis, no cultivo do híbrido Miltonia regnellii Rchb. f. X Oncidium concolor Hook., com oito meses de idade, durante um ano.

\section{Referências}

ASSIS, A. M. ; COLOMBO, L. A.; FARIA, R.T.; FONSECA, I. C. B. Longevidade pós-colheita de pseudobulbos com flores de Dendrobium nobile (Orchidaceae). Revista Brasileira de Horticultura Ornamental, Campinas, v. 9, n. 1, p. 85-87, 2003.

ASSIS, A. M. ; FARIA, R.T.; COLOMBO, L. A.; CARVALHO, J. F. R. P. Utilização de substratos à base de coco no cultivo de Dendrobium nobile Lindl. (Orchidaceae). Acta Scientiarum, Maringá, v. 27, n. 2, p. 255-260, abr./jun. 2005.

BASSO, F. M.; FARIA, R.T. Utilização de diferentes substratos no cultivo de Laelia lundii (Orchidaceae) visando a preservação do xaxim. In: MOSTRA ACADÊMICA DE TRABALHOS DE AGRONOMIA, 6., 2002, Londrina. Anais... Londrina: UEL, 2002, p. 48.

BEZERRA, F. C.; ROSA, M. F.; BRÍGIDO, A. K. L.; NORÕES, E. R. V. Utilização de pó de coco como substrato de enraizamento para estacas de crisântemo. Revista Brasileira de Horticultura Ornamental, Campinas, v. 7, n. 2, p.129-134, 2001.
BOSA, N.; CALVETE, E. O.; KLEIN, V. A.; SUZIN, M. Crescimento de gipsofila em diferentes substratos. Hoticultura Brasileira, Brasília, v. 21, n. 3, p. 514-519, 2003.

COLOMBO, L. A.; FARIA, R.T.; ASSIS, A.M.; FONSECA, I. C. B. Aclimatização de um híbrido de Cattleya em substratos de origem vegetal sob dois sistemas de irrigação. Acta Scientiarum, Maringá, v. 27, n. 1, p. 145-150, jan./mar. 2005.

DEMATTÊ, M. E. S. P. Cultivo de Tillandsia gardneri Lindl. em diferentes substratos. In: CONGRESSO BRASILEIRO DE FLORICULTURA E PLANTAS ORNAMENTAIS, 13., 2001, São Paulo. Resumos...São Paulo: SBFPO, 2001. p. 118.

FARIA, R. T.; REGO, L.V.; BERNARDI, A.; MOLINARI, H. Performance of diferents genotyps of brazilian orchid cultivation in alternatives substrates. Brazilian Archives of Biology and Technology, Curitiba, V. 44, n. 4, p. 337-342, 2001.

FERMINO, M. H. Aproveitamento de resíduos industriais e agrícolas como alternativas de substratos hortícolas. 1996. 90 f. Dissertação (Mestrado em Fitotecnia) Programa de Pós-Graduação em Agronomia. Faculdade de Agronomia, Universidade Federal do Rio Grande do Sul, Porto Alegre, 1996.

GALDIANO JÚNIOR, R. F.; MORAES; M.; LEMOS, E. G. M. . Utilização de substratos alternativos ao xaxim para o crescimento de um híbrido de Cattleya Lindley (Orchidaceae). In: CONGRESSO BRASILEIRO DE FLORICULTURA E PLANTAS ORNAMENTAIS, 16.; CONGRESSO BRASILEIRO DE CULTURA DE TECIDOS DE PLANTAS, 3.; SIMPÓSIO DE PLANTAS ORNAMENTAIS NATIVAS, 1., 2007, Goiânia. Resumos ... Goiânia: SBFPO, 2007. v. 13, p. 1675.

GUERRINI, I. A.; TRIGUEIRO, R. M. Atributos físicos e químicos de substratos compostos por biossólidos e casca de arroz carbonizada. Revista Brasileira de Ciência do Solo, Viçosa, v. 28, n. 6, p. 1069-1076, 2004.

HUMBER, G. Onde se desenvolvem as orquídeas. 5. ed. Rio de Janeiro: Expressão e Cultura, 1994. p. 14-16.

KÄMPF, A. N. Seleção de materiais para uso como substratos. In: ENCONTRO NACIONAL SOBRE SUBSTRATOS PARAPLANTAS, 1., 1999, Porto Alegre. Anais... Porto Alegre: Gênesis, 1999. p. 139-145.

KÄMPF, A. N. Produção comercial de plantas ornamentais. Guaíba: Agropecuária, 2000. 254p.

LACERDA, M. R. B.; PASSOS, M. A. A.; RODRIGUES, J. J. V.; BARRETO, L. P. Características físicas e químicas 
de substratos à base de pó de coco e resíduo de sisal para produção de mudas de sabiá (Mimosa caesalpiniaefolia Benth). Revista Árvore, Viçosa, v. 30, n. 2, p. 163-170, mar./abr. 2006.

MENEGUCE, B.; OLIVEIRA, R. B. D.; FARIA, R. T. Propagação vegetativa de Epidendrum ibaguense Lindl. (Orchidaceae) em substratos alternativos ao xaxim. Semina: Ciências Agrárias, Londrina, v. 25, n. 2, p. 101106, 2004.

MORAES, L. M.; CAVALCANTE, L. C. D.; FARIA, R. T. Substratos para aclimatização de plântulas de Dendrobium nobile Lindl. (Orchidaceae) propagadas in vitro. Acta Scientiarum, Maringá, v. 24, n. 5, p. $1397-$ 1400, 2002.

NEGRÃO, L. C. P.; URBAN, M. L. P. Álcool como "Commodity" Internacional. Economia \& Energia, Belo Horizonte, v. 8, n. 47, jan. 2005. Disponível em: <http:// www.ecen.com/eee47/eee47p/ecen_47p.htm>. Acesso em: 17 maio 2007.

PASCAL, M.; ARAUJO, A. G. ; RODRIGUES, V. A.; OLIVEIRA, A. C.. Cultivo de orquídeas. Informe Agropecuário, Belo Horizonte, v. 26, n. 227, p. 85-94, 2005.

REGO, L.V.; BERNARDI, A.; TAKAHASHI, L. S. A.; FARIA, R. T. Desenvolvimento vegetativo de genótipos de orquídeas brasileiras em substratos alternativos ao xaxim. Revista Brasileira de Horticultura Ornamental, Campinas, v. 6, n.1/2, p.75-79, 2000.

ROSA, M. F.; BEZERRA, F. C.; ARAÚJO, F. B. S.; NORÕES, E. R. V. Utilização do pó da casca de coco verde na germinação de alface hidropônico. Horticultura Brasileira, Brasilia, v. 19, n. 2, p. 294, 2001.

ROSA, M. F.; BEZERRA, F. C.; CORREIA, D.; SANTOS, F.J. S.; ABREU, F.A. P. ; FURTADO, A. A. L.; BRÍGIDO, A. K. L.; NORÕES, E. R.V. Utilização de coco como substrato agrícola. Fortaleza: Embrapa Agroindústria Tropical, 2002. 24p.

SOUZA, N. A. JASMIM, J. Uso de casca de coco em substrato e tutor para o cultivo de singônio. In: CONGRESSO BRASILEIRO DE FLORICULTURA E PLANTAS ORNAMENTAIS, 13., 2001, São Paulo. Resumos... São Paulo: SBFPO, 2001. p. 108.

SILVA, F. S. C.; SILVA, S. P. C. O substrato na cultura das orquídeas, sua importância, seu envelhecimento. Revista Oficial da Orquidário, Rio de Janeiro, v. 11, n. 1, p. 3-10, 1997.

SILVA, W. Cultivo de orquideas o Brasil. São Paulo: Nobel, 1986.

STEGANI, V. Aclimatização de orquídea utilizando como substrato pó de bagaço de cana-de-açúcar. 2006. $32 \mathrm{f}$. Dissertação (Mestrado em Agronomia) -Universidade Estadual de Maringá, Maringá, 2006.

SUTTLEWORTH, F. S.; ZIMMERMAN, H. S.; DILLON, G. W.; SMITH, E. W. Orchids. São Francisco: Western Publishing Company, 1970.

YAMAKAMI, J. K.: FARIA, R. T.; ASSIS, A. M.; OLIVEIRA, L. V. R. Cultivo de Cattleya Lindley (Orchidaceae) em substratos alternativos ao xaxim. Acta Scientiarum, Maringá, v. 28, n. 4, p. 523-526, out./dez. 2006. 